\title{
Experimental setup for investigating the dynamics of the ascent of a cluster of bubbles in a liquid
}

\author{
Vladimir Arkhipov ${ }^{1}$, Sergey Basalaev ${ }^{1, *}$, Sergey Orlov ${ }^{1}$, and Sergey Polenchuk ${ }^{1}$ \\ ${ }^{1}$ National Research Tomsk State University, 634050 Tomsk, Russia
}

\begin{abstract}
There is a scheme of experimental setup providing preparation of the compact cluster of monodispersed bubbles of the given diameter presented in the work. Further, in this research we have calculated setup parameters that are necessary for preparation of the compact air bubble cluster in glycerin at room temperature.
\end{abstract}

\section{Introduction}

The behavior of fluid containing bubbles is distinctly different from behavior of homogeneous fluids under different physical and physical-chemical influence. These differences are abundantly used in industry - boiling, heat transfer in the two-phase mediums, cavitation, foaming, flotation. There is a question of the bubble cluster of given size generation in a number of problems, in particular, while researching an ignition of fluid electric discharge in fluids by way of specially created cavitational bubbles [1], while researching surfactants and acoustic waves on dynamics of bubble clusters [2-3].

In practice of the experimental studies there are setup relating to aeration devices, aim to gas introduction to the liquid fluid. The carried out analysis devices work [4-5] showed that setups are intended for creation of the continuous flow of bubbles in fluid. The [6] disadvantage is impossibility of cluster generation from monodispersed bubbles of millimetric sizes and also complexity of the setups realizing these methods. The method [7] does not allow to generate a bubble cluster of controlled form.

In this paper, the experimental scheme of setup allowing to provide a compact cluster of monodispersed bubbles of the given diameter was described.

\section{The scheme of the experimental setup}

The experimental setup for generation of a compact cluster of monodispersed bubbles [8] is presented in Fig. 1. The setup includes collector 3 that placed in a lower part of receiver 1 with fluid 2 with gas-permeable upper cover 4 , connected by branch pipe 5 with a source of compressed gas. Receiver 1 is executed in the form of a cuvette with plane-parallel walls

\footnotetext{
* Corresponding author: tarm@niipmm.tsu.ru
} 
from optical glass of $0.3 \times 0.3 \times 0.6 \mathrm{~m}$ in size for visualization possibility of upfloat process of bubbles cluster.

In upper cover 4 of collector 3 are executed in its center and for equidistant concentric circles of perforation in which central 6 and peripheral tubes 7 of identical diameter are established which height that is identical to the tubes located on each circle and linearly decreases with increase of circle radius. Injection medical needles are used as tubes.

Cylinder 8 with the compressed gas, connected through low pressure reducer 9 and also through high pressure reducer 11 and electropneumatic valve 13 with branch pipe 5 of collector 3 is used as a source of gas.

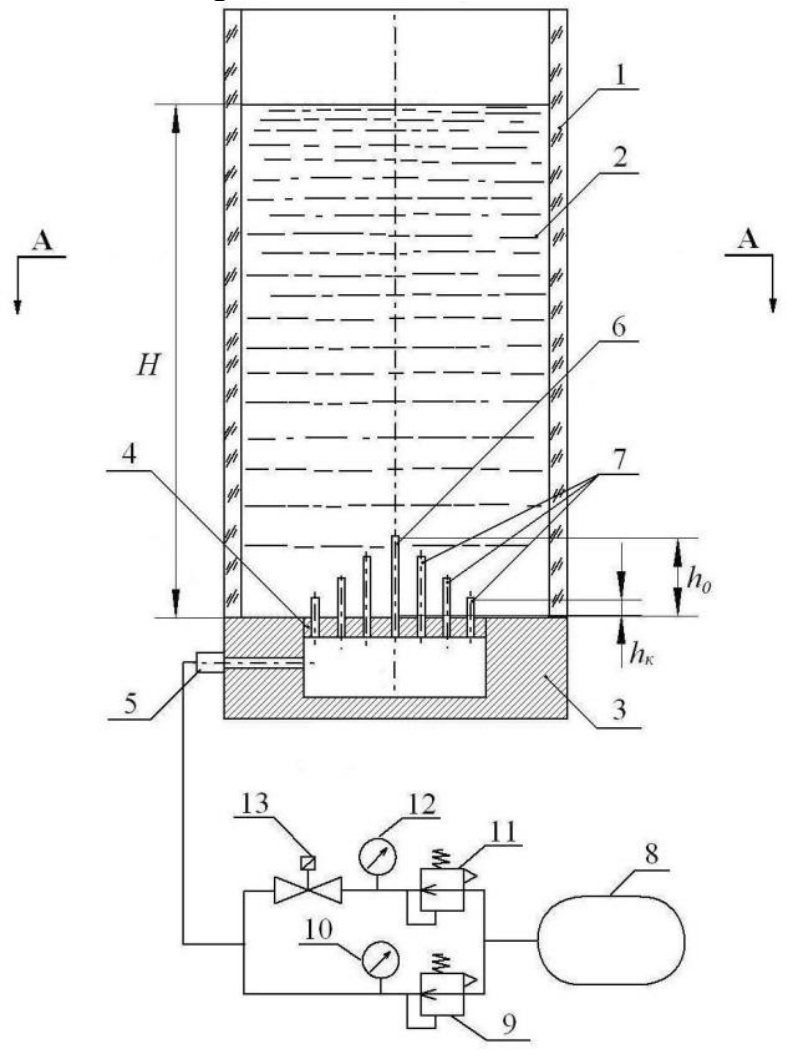

Fig. 1. The scheme of the experimental setup for creation of a compact cluster of monodispersed bubbles.

Work of the setup is carried out as follows. The constant pressure $p_{\min }$ controlled by a pressure gage 10 is settled by means of the reducer 9 , it hinders fluid 2 flowing through tubes 6, 7 into the collector 3. By means of high pressure reducer 11 and the electropneumatic valve 13 the compressed gas is pulsewise supplied under the pressure $p_{\max }$ through the branch pipe 5 into the collector 3 . Gas from the collector 3 through tubes 6,7 in the form of bubbles comes to ambient liquid 2. After bubbles departure from tubes 6,7 in liquid 2 the compact cluster of spherical shape bubbles floating up is formed.

\section{Results of the experimental study}

Let's consider the results of the compact cluster preparation of air monodispersed bubbles in glycerin at room temperature. Parameters of the device, necessary for calculations, are specified in table 1 , where $D$ - the required diameter of the formed bubble; $d$ - inside 
diameter of a tube; $h_{0}$ - height of the central tube; $R$ - radius of the upper cover of a collector; $H$ - height of fluid column in the receiver over the collector upper cover.

Table 1. Parameters of a setup.

\begin{tabular}{|c|c|c|c|c|}
\hline$D(\mathrm{~mm})$ & $d(\mathrm{~mm})$ & $h_{0}(\mathrm{~mm})$ & $R,(\mathrm{~mm})$ & $H,(\mathrm{~mm})$ \\
\hline 5 & 0.26 & 30 & 64 & 500 \\
\hline
\end{tabular}

Physical characteristics of air and glycerol [9] at a temperature of $20^{\circ} \mathrm{C}$, are shown in table 2, where $\rho$-density; $p_{\text {atm }}$ - atmospheric pressure; $\sigma$ - coefficient of superficial liquid tension.

Table 2. Physical characteristics of air and glycerin.

\begin{tabular}{|c|c|c|}
\hline Parameter & Air & Glycerin \\
\hline$\rho, \mathrm{kg} / \mathrm{m}^{3}$ & 1.205 & 1260 \\
\hline$\mu, \mathrm{Pa} \cdot \mathrm{c}$ & $1.808 \cdot 10^{-5}$ & - \\
\hline$\sigma, \mathrm{H} / \mathrm{m}$ & - & $63 \cdot 10^{-3}$ \\
\hline
\end{tabular}

Now we are calculating parameters of the setup. For determination of the tubes height $h_{i}$ located on circle radius $r_{i}$, we consider similar triangles of $\mathrm{ABC}$ and $\mathrm{AB}_{1} \mathrm{C}_{1}$ (Fig. 2).

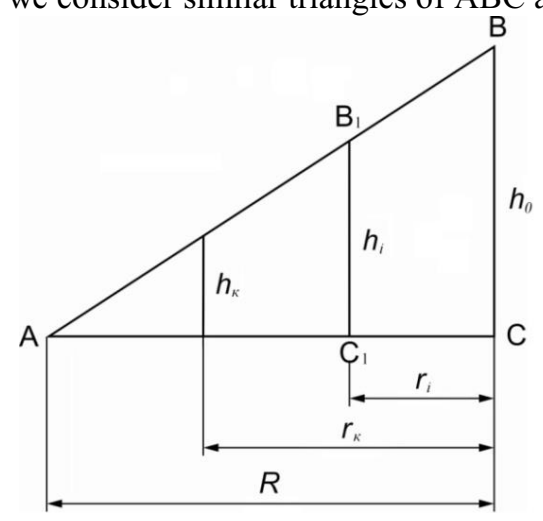

Fig. 2. Similar triangles of $A B C$ and $A B_{1} C_{1}$.

From the similarity condition follows:

$$
\frac{B C}{A C}=\frac{B_{1} C_{1}}{A C_{1}} .
$$

From Fig. 1 follows:

$$
B C=h_{0}, A C=R, B_{1} C_{1}=h_{i}, A C_{1}=R-r_{i},
$$

where $h_{\mathrm{i}}$, tube height of located on radius $r_{i}(i=1,2, \ldots, \kappa)$.

Substituting (2) in (1), we are getting: $h_{0} / R=h_{i} /\left(R-r_{i}\right)$, from here follows the ratio:

$$
h_{i}=h_{0}\left(1-\frac{r_{i}}{R}\right) .
$$

The ratio (3) provides linear reduction of tubes height from $h_{0}$ to $h_{k}$ with increase in radius of circle $r_{i}$. The results of calculations of tubes height located on a circle with radius $r_{i}$ are provided in table 3. 
Table 3. Height of the tubes located on a circle radius $r_{i}$.

\begin{tabular}{|c|c|c|c|}
\hline$i$ & 1 & 2 & 3 \\
\hline$r_{i}(\mathrm{~mm})$ & 16 & 32 & 48 \\
\hline$h_{i}(\mathrm{~mm})$ & 22.5 & 15 & 7.5 \\
\hline
\end{tabular}

Under a pressure in the collector equal to the minimum pressure quantity of gas $p_{\min }$, gas shouldn't come into liquid through tubes. It is provided under the following condition:

$$
p_{\min }<p_{\text {atm }}+p_{h}
$$

where $p_{h}=\rho g h$, hydrostatic pressure; $\rho$, liquid density; $g$, acceleration of gravity; $h$, liquid column height over discharge ring of the tube.

The smallest hydrostatic pressure is implemented for the central tube with height $h_{0}$ for which $h=H-h_{0}$. From (4) follows:

$$
p_{\min }<p_{\text {atm }}+\rho g\left(H-h_{0}\right) .
$$

During the development of the setup we experimentally get more specific condition (5):

$$
p_{\text {min }}=p_{\text {atm }}+0.8 \rho g\left(H-h_{0}\right)=105954 \mathrm{~Pa} .
$$

While performing ratio (6) gas doesn't come into liquid through the central tube and also through the tubes located on concentric circles, because hydrostatic pressure is higher for them, than for the central tube.

Under a pressure in the collector equal to the maximum pressure quantity of gas $p_{\max }$, gas should come through the tubes into liquid. It is provided under the following condition:

$$
p_{\max }>p_{\mathrm{atm}}+p_{h}
$$

The largest hydrostatic pressure is implemented for peripheral tubes with height $h_{k}$ for which $h=H-h_{k}$. From (7) follows:

$$
p_{\max }>p_{\text {atm }}+\rho g\left(H-h_{k}\right) .
$$

During the development of the setup we experimentally get more specific condition (8):

$$
p_{\max }=p_{\mathrm{atm}}+1.2 \rho g\left(H-h_{k}\right)=108611 \mathrm{~Pa} .
$$

While performing ratio (9) gas bubbles come into liquid through the tubes located on peripheral circle radius $r_{k}$ and also through other tubes, because hydrostatic pressure is less for them, than for peripheral tubes.

For determination of impulse duration $\tau$ of the electropneumatic valve openning we will consider the formula of gas consumption through the tube [10]:

$$
Q=\varphi S \sqrt{\frac{2 \Delta p}{\rho_{g}}}
$$

where $Q$, volume consumption of gas; $\varphi$ - consumption coefficient; $S=\pi d^{3} / 4$, tube crosssectional area; $\Delta p=0.2 \rho g\left(H-h_{k}\right)$, pressure difference on a tube; $\rho_{\mathrm{g}}$, gas density.

The volume of the gas coming to liquid during time $\tau$, is defined by a formula: 


$$
V_{g}=Q \cdot \tau=\varphi S \tau \sqrt{\frac{2 \Delta p}{\rho_{g}}}
$$

The input of gas portion with volume $V_{g}$ forms a bubble which volume is equal to the volume of the entered gas:

$$
V=\frac{\pi D^{3}}{6}=V_{g}
$$

From (11), (12) the ratio for definition follows $\tau$ :

$$
\tau=\frac{\pi D^{3}}{6 \varphi S} \sqrt{\frac{\rho_{g}}{2 \Delta p}}=\frac{2}{3} \frac{D^{3}}{\varphi d^{2}} \sqrt{\frac{\rho_{g}}{0.4 \rho g\left(H-h_{k}\right)}}=0.055 \mathrm{~s}=55 \mathrm{~ms}
$$

When calculating $\tau$ the value of the consumption coefficient $\varphi=0.5$ is defined according to $[10,11]$.

For the calculated parameters of the setup $\left(p_{\min }=105954 \mathrm{~Pa} ; p_{\max }=108611 \mathrm{~Pa}\right.$; $\tau=0.055 \mathrm{~s}$ ) there was a series of experiments conducted, for preparation of the monodispersed bubbles compact cluster and the analysis of dynamics of its upfloat in nonperfect fluid. The video frames of upfloating of the monodispersed bubbles compact cluster received in two perpendicular planes are provided on Fig. 3. Diameter of bubbles of $D \approx 5 \mathrm{~mm}$ received experimentally.
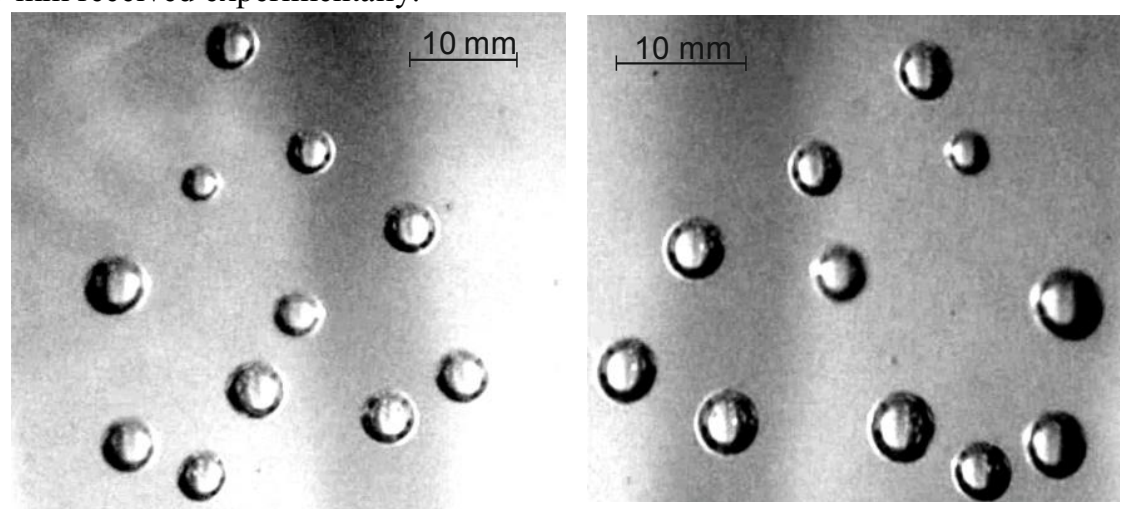

Fig. 3. The video frames of upfloating of the monodispersed bubbles compact cluster.

\section{Conclusion}

- The modified scheme of experimental setup providing the compact cluster preparation of monodispersed bubbles of the given diameter is presented in the work.

- An increase in the ascent rate of a cluster of bubbles was found, compared to the velocity of a single bubble in a pure liquid.

- Performing perforations in the top cover of the collector at its center and along equidistant concentric circles makes it possible to obtain an axisymmetric bubble cluster.

- The use of microtubules of the same diameter, installed in perforations, provides the formation of monodisperse bubbles.

- The use of microtubules of equal height located along each of the concentric circles ensures the simultaneous formation of a "ring" of bubbles for each of the circles. 
- The linear decrease in the height of the tubes located on the circles, with an increase in the radius of the circle, ensures the sequential formation of each "ring" of bubbles with the same time lag as they move away from the center of the collector cover. This allows us to obtain a compact cluster with a uniform spatial distribution of bubbles.

This study was supported by the Russian Science Foundation (Project No. 15-19-10014).

\section{References}

1. A. P. Drozhzhin, S. M. Korobeynikov, B. C. Teslenko, Scientific bulletin of NGTU 2 (2003) [in Russian]

2. V. G. Levich, Physical and chemical hydrodynamics (Fizmatgiz, Moscow, 1959) [in Russian]

3. V. A. Arkhipov, I. M. Vasenin, A. S. Usanina, Fl. Dyn. 51 (2), (2016)

4. M. Hanok, Aeration device for introduction of gas bubbles on liquid medium, Patent RU 2491116, (Int.Cl.: B01F 3/04, B01F 13/00, 2013 ) [in Russian]

5. II. Sheaffer, R. John Aeration device for aquatic environments Patent WO 2016003926 (Int.Cl.: B01F 3/04262, C02F 1/74, 2016)

6. S. Naohiro, A. Keita, S. Toshihiko, Ultras. 77 (2017)

7. E. O. Oksman. Bubble-generating aerator, Patent US 3970731 (Int.Cl.: B01F 3/04, C02F 3/20, 1976)

8. V. A. Arkhipov, A. S. Usanina, K. G. Perfilieva, S. A. Basalaev, S. N. Polenchuk, V. I. Romandin. The setup for generation of a compact cluster of monodispersed bubbles. The application on patent RU 2017142492 (Int.Cl.: B01F 3/04, C02F 3/20, 2017) [in Russian]

9. F. V. Nevolin. Chemistry and technology of glycerin production (Chemistry, Moscow, 1954) [in Russian]

10. P. P. Kremlin. Flowmeters and counters of quantity Reference book 4th edition, revised and enlarged (Machine manufacturing, Leningrad, 1989) [in Russian].

11. S. Goldaev, S. Basalaev, K. Afanasyev, MATEC Web Conf. 110, 01033 (2017) 